\title{
Ingeniería del mantenimiento industrial y gestión del conocimiento. Mejora en la eficiencia de las empresas
}

\author{
Industrial Maintenance Engineering and \\ Knowledge Management. Improvement in \\ Business Efficiency
}

\author{
Francisco Javier Cárcel-Carrasco* \\ Universidad Politécnica de Valencia, Valencia, España. \\ FECha DE ENTREGA: 10 DE NOVIEMBRE DE 2014 \\ FECHA DE EVALUACIÓN: 27 DE ENERO DE 2015 \\ FECHA DE APROBACIÓN: 27 DE ENERO DE 2015
}

\begin{abstract}
Resumen En este artículo se muestra el nivel estratégico que, para las empresas con activos físicos, supone una adecuada gestión del conocimiento en la ingeniería del mantenimiento industrial. Por las peculiaridades propias que se dan normalmente en este tipo de actividad fundamental de la empresa, el conocimiento de los operarios y técnicos que operan en las áreas de mantenimiento está fuertemente basado en su experiencia (fuerte componente tácito), difícil de medir y articular, y sin embargo, en numerosas ocasiones, esta rotura de la información-conocimiento, puede suponer un alto coste para la empresa, muchas veces asumido como algo que afrontar, debido al incremento de tiempos de parada de producción y servicios, perdidas de eficiencia energética, o tiempo de acoplamiento de nuevo personal a estas áreas. Este artículo muestra una reseña sobre dos libros de investigación que tratan sobre este tema, publicados tras una investigación de campo aplicada en empresas industriales reales europeas, que marcan las consecuencias de disponer el conocimiento en islas, así como las mejoras medidas cuando se produce una adecuada gestión del conocimiento.
\end{abstract}

Abstract This article demonstrates the strategic level involving management of knowledge in industrial maintenance engineering for businesses with physical assets. The peculiarities that occur normally in this type of basic activity of the company, knowledge of operators and technicians working in the areas of maintenance is strongly based on your experience (strong tacit component), difficult to measure and articulate, however, on numerous occasions, this break of information-knowledge, can signify a high cost for the companies taken as something to deal with, due to the increase of downtimes of production and services, loss of efficiency,

* fracarc1@csa.upv.es 
or time of coupling of new personnel to these areas. This article shows a review of two books of research dealing with this topic, published after a field investigation applied to real industrial companies that make the consequences of having knowledge on islands, as well as measures improvements when there is an adequate knowledge management.

Palabras Clave: eficiencia energética, mantenimiento industrial, gestión del conocimiento, industria agroalimentaria.

Keywords: energy efficiency, industrial maintenance, management of knowledge, food industry.

\section{Introducción}

La ingeniería del mantenimiento industrial requiere de conocimientos técnicos muy específicos, un alto requerimiento de experiencia del personal que lo desenvuelve, con un alto componente de conocimiento tácito, y con poca tradición en transcribir las experiencias que se producen. La adecuada gestión del conocimiento y la aplicación del conocimiento adquirido en las actividades rutinarias de mantenimiento en la empresa, y su mejora, puede ser observado como un factor o proceso importante que puede influir positivamente en diversas acciones que afectan estratégicamente a toda la empresa, y entre ellas, las acciones que afectan a la fiabilidad, operación en explotación y la eficiencia energética.

Aunque existen muchos estudios teóricos sobre cómo aplicar técnicas de gestión del conocimiento en organizaciones o en empresas de carácter general, en la literatura actual no existen estudios empíricos de su aplicación a una organización de mantenimiento industrial de una empresa, enfocados a mejorar sus acciones estratégicas fundamentales (la mantenibilidad, la fiabilidad y la eficiencia energética).

Durante un periodo de dos años se realizó un estudio exploratorio y de campo para ver la incidencia de la adecuada gestión del conocimiento en empresas industriales europeas con importante equipo humano en las áreas de mantenimiento operativo. Posteriormente tras los datos de campo obtenidos, se planteó y aplicó un modelo de mantenimiento industrial basado en la gestión del conocimiento en una empresa de primer orden, obteniéndose resultados que fueron contrastados tras un periodo continuo de tres años. Del resultado de estas investigaciones se publicaron dos libros de investigación en la editorial científica Omnia Science [1] [2] (publicados en open access, para descarga libre, por parte de cualquier investigador interesado) (Figura 1). Estos libros son "La gestión del conocimiento en la ingeniería del mantenimiento industrial: Investigación sobre la incidencia en sus actividades estratégicas". Omnia Science, 2014. ISSN 978-84-941872-7-8 y "Planteamiento de un modelo de mantenimiento industrial basado en técnicas de gestión del conocimiento". Omnia Science, 2014. ISSN 978-84-941872-8-5.

En los puntos siguientes del artículo, se comentan los aspectos fundamentales de estas publicaciones objeto de la investigación de campo en esta área. 


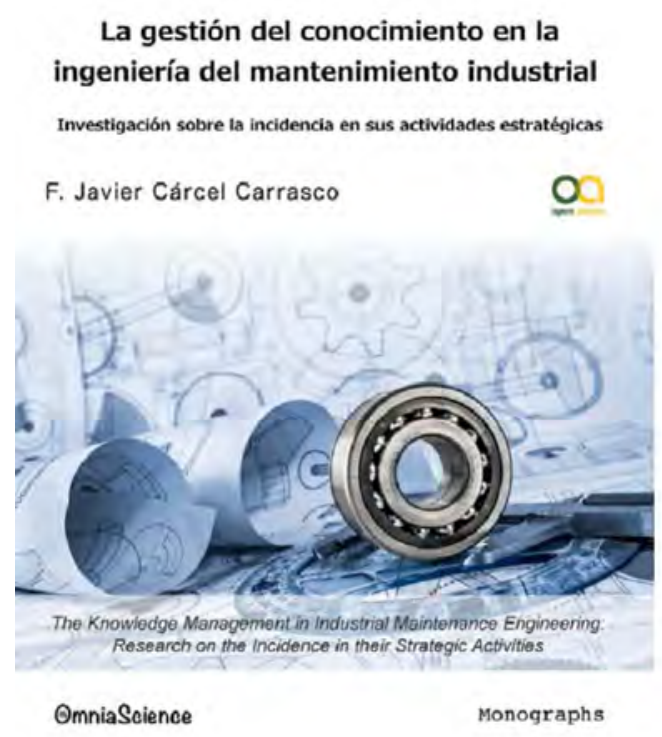

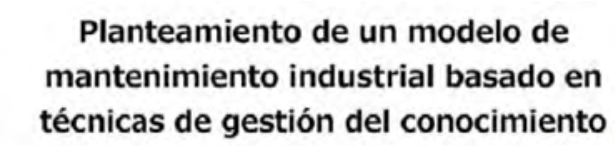

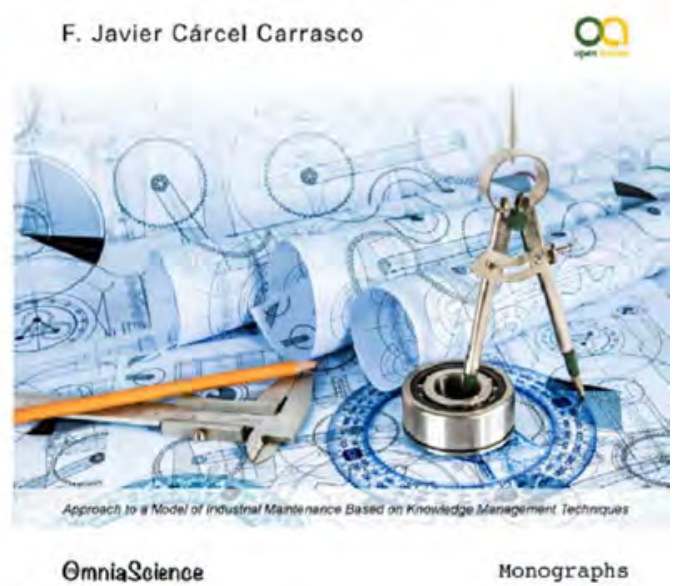

Figura 1. Portadas de los libros de investigación publicados en referencia a la gestión del conocimiento en la ingeniería del mantenimiento industrial.

\section{La gestión del conocimiento en la ingeniería del mantenimiento industrial}

En el libro de investigación "La gestión del conocimiento en la ingeniería del mantenimiento industrial: Investigación sobre la incidencia en sus actividades estratégicas" [1], se estudian e identifican en dos etapas las características del mantenimiento industrial y la gestión del conocimiento. La primera etapa, está orientada a la identificación del estado de la situación del mantenimiento, los principios y técnicas de la gestión del conocimiento, y la descripción de los modelos organizativos de mantenimiento industrial y sus misiones estratégicas fundamentales en relación al conocimiento y la experiencia, estableciendo la evolución y el estado del arte de esta materia y los mecanismos en relación a la transmisión de la información, y en especial, al conocimiento tácito.

En una segunda etapa, se analizan mediante estudios cualitativos con entrevistas, cuestionarios y encuestas preparadas y analizadas en un entorno industrial, los aspectos estratégicos del mantenimiento en relación a la fiabilidad (o confiabilidad), la mantenibilidad, la eficiencia energética y la operatividad en explotación, con el fin de establecer y confirmar los mecanismos de captación, generación, transmisión y utilización del conocimiento estratégico que se usan en la propia organización de mantenimiento.

El proceso de gestión del conocimiento integrado básicamente, por la generación, la codificación, la transferencia y la utilización del conocimiento aplicado a la actividad táctica del mantenimiento, puede tener un enfoque kantiano en el 
cual interactúan personas, instalaciones y entorno (Figura 2), en el cual deben ser estudiadas todas las variables en conjunto.

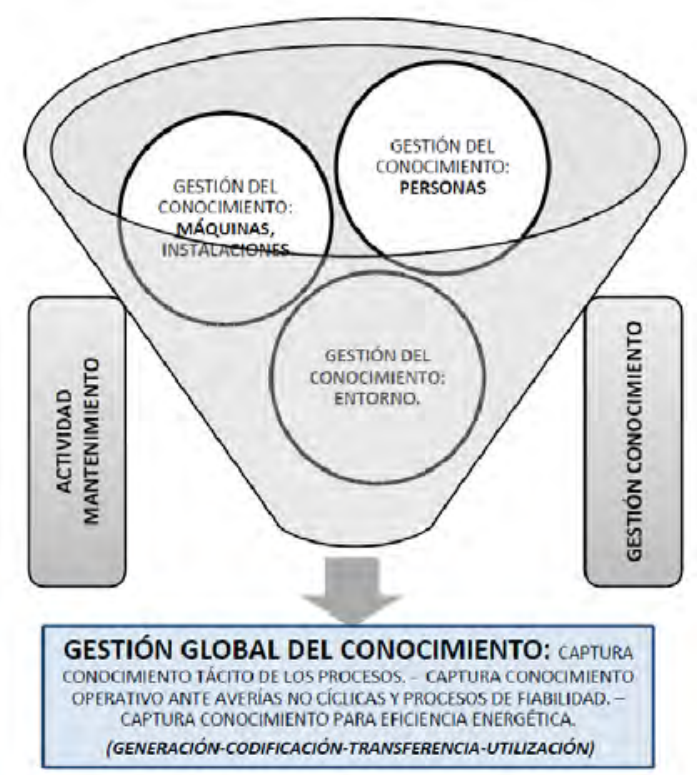

Figura 2. Enfoque kantiano de la actividad de mantenimiento.

Hay que tener en cuenta los problemas más frecuentes y críticos, en relación al conocimiento tácito y la gestión del conocimiento con los que los especialistas y técnicos de mantenimiento se encuentran, varios. A continuación se listan los que se deben tener en cuenta en este tipo de organizaciones.

- Cambios de personal de la plantilla (pérdida del conocimiento de la persona que causa baja).

- Poca experiencia de los operarios (tiempo en formar conocimiento para ser operativo en el entorno).

- Falta de información de medidas a tomar y pasos a seguir ante ciertas averías o incidencias (Conocimiento ante actuaciones no registradas).

- Dependencia del conocimiento y experiencia tácita de los operarios (Conocimiento que hace cautiva a la empresa).

- Históricos de avería y análisis de causas imperfectos (Conocimiento incompleto o mal documentado).

- Desorganización de la información acerca de las instalaciones (Conocimiento explícito mal organizado o no actualizado (planimetría, manuales, procedimientos).

- Carencia de sistemas de aprendizaje y reciclaje del personal (Adquisición del conocimiento útil y aplicado).

- Actuación ante averías críticas, de emergencia o no cíclicas (conocimiento crítico de graves efectos económicos). 
Todos estos problemas fundamentales, aunque simples en definición y de apariencia banal, pueden tener graves consecuencias en el proceso productivo que afectarán sin duda a la empresa, aunque muchas veces asumidos. Son problemas complejos de tratar y procesar, dada la alta dependencia del factor humano, requiere de un compromiso global con unas dotaciones de medios y un seguimiento a largo plazo, mostrando con ello la dificultad de las empresas en la aplicación de estrategias globales de gestión del mantenimiento y su conocimiento estratégico.

Se confirma en el presente estudio, que la transcendencia de una adecuada gestión del conocimiento puede tener sobre las actividades estratégicas fundamentales de mantenimiento confirmadas por todo el personal entrevistado (fiabilidad, mantenibilidad, eficiencia energética y operación/explotación). En la figura 3, se extraen las principales características observadas en función de las actividades estratégicas, y que redundan en la eficiencia de la actividad de la empresa.

Se reconoce, que una mejora en la gestión de la información y conocimiento, redunda positivamente en todas esas acciones, y en especial en la resolución de grandes averías, o fallos no cíclicos espaciados en el tiempo y normalmente no registrada su actuación.

En cuanto a las herramientas que pueden ser utilizadas para recabar información estratégica que ayude a mejorar la gestión del conocimiento, normalmente son poco utilizadas en todos los ambientes de mantenimiento. Se reconoce la poca utilización de auditorías en las acciones internas, los mapas de información y conocimiento, realizándose diagramas de criticidad sólo en determinadas instalaciones o equipamiento fundamental para la actividad de la empresa.

Se detecta un mayor uso de las reuniones informales como medio de generación y transferencia del conocimiento, sobre todo, entre los grupos de técnicos operativos, con una menor cultura organizativa que los mandos o jefes de mantenimiento.

\section{Planteamiento de un modelo de gestión del conocimiento en la ingeniería del mantenimiento industrial}

Tras el estudio preliminar donde se obtienen los datos que marcan las características de la relación "mantenimiento industrial-Gestión del conocimiento" cuyos datos y conclusiones son indicados en el libro anterior, se planteó un modelo aplicado experimental sobre una industria europea de primer nivel en un periodo de dos años. El modelo y los resultados están indicados en el libro de investigación "Planteamiento de un modelo de mantenimiento industrial basado en técnicas de gestión del conocimiento" [2], donde se indican cómo se ha abordado y que resultados se han obtenido. Algunas metas que persigue la investigación son las siguientes:

- Estudiar y analizar los flujos de conocimiento (en especial el tácito), investigando los mapas de conocimiento que afectan a los fines tácticos de la ingeniería de mantenimiento. 


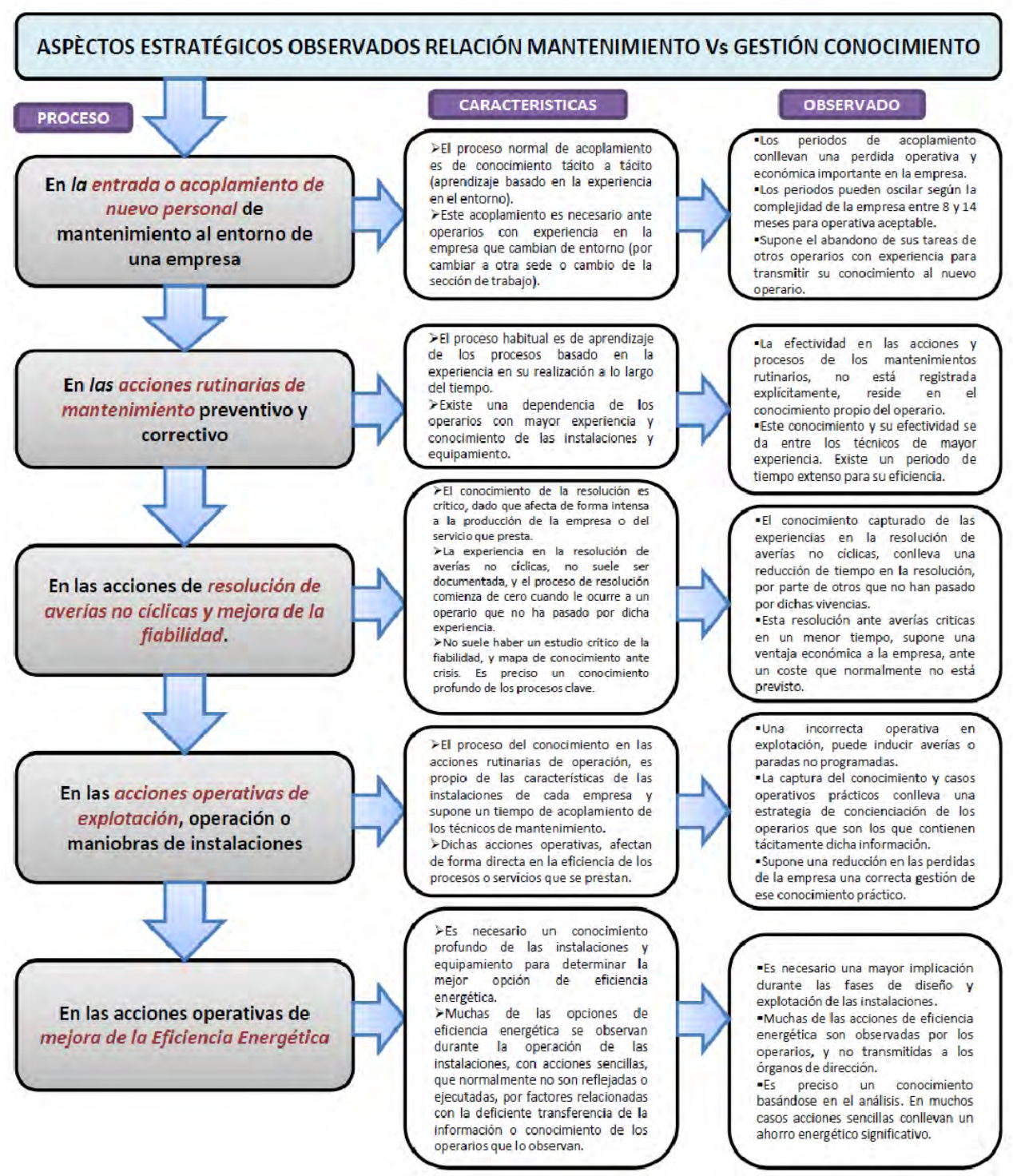

Figura 3. Aspectos estratégicos del mantenimiento y su relación con la gestión del conocimiento.

- Mejorar las condiciones de transmisión del conocimiento en la actividad de mantenimiento, que produzcan una mayor rapidez en el acoplamiento operativo de nuevo personal, o de técnicos pertenecientes a otras áreas.

- Unir las técnicas y herramientas operativas de la actividad de mantenimiento con la adecuada gestión del conocimiento, para mejora de la fiabilidad y respuesta ante fallo de los sistemas de la empresa.

- Unir las técnicas y herramientas operativas de la actividad de mantenimiento con la adecuada gestión del conocimiento, para mejora de la eficiencia energética de los sistemas técnicos de la empresa. 
- Unir las técnicas y herramientas operativas de la actividad de mantenimiento con la adecuada gestión del conocimiento, para mejora de la mantenibilidad de la empresa.

- Utilizar las técnicas de gestión de conocimiento como sistema de autoaprendizaje, decisión y sistema de reciclaje del personal, tanto de ubicación y características de las instalaciones, como de tipos de fallos y soluciones a adoptar ante fallos en las mismas.

- Utilizar la distribución del conocimiento en la adecuada planificación y control del proceso de mejora de las actuaciones de mantenimiento

Todos los objetivos arriba detallados están encaminados a conseguir un fin primordial: una efectiva acción de la actividad de mantenimiento por utilización de la gestión del conocimiento.

La evolución hacia un modelo de gestión del conocimiento aplicado al mantenimiento industrial debe pasar por tres fases fundamentales, desde la identificación del conocimiento intangible y tangible útil, detentando las barreras para su implantación, la transformación de lo intangible en tangible, finalizando en los procesos para la generación, producción y utilización del conocimiento (Figura $4)$.

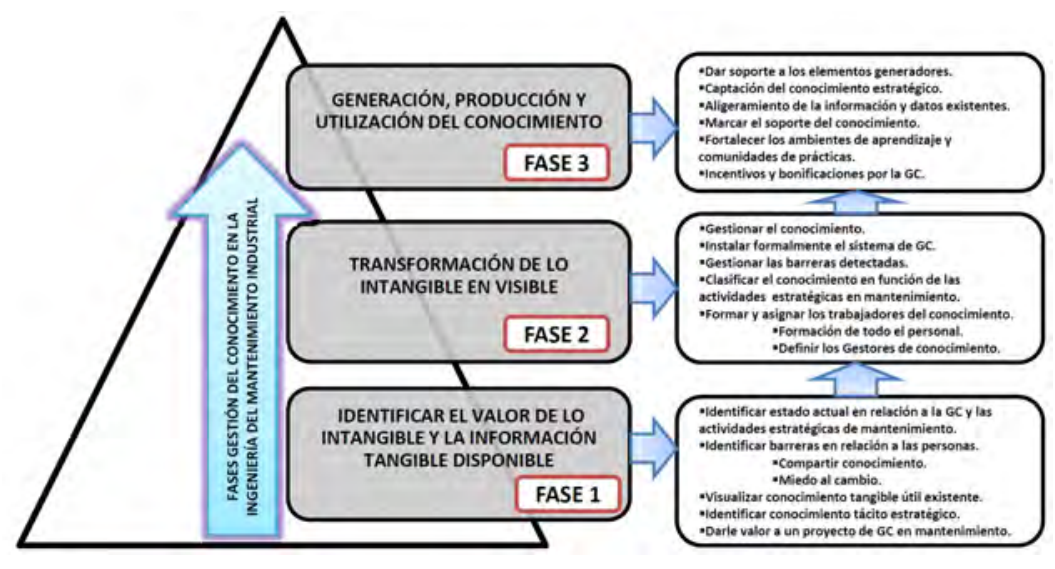

Figura 4. Fases de la evolución de la gestión del conocimiento en mantenimiento industrial. Fuente: Elaboración propia.

En una primera fase fundamental, se identifica el valor del conocimiento intangible (conocimiento tácito), así como la situación de la información tangible existente (planimetría, memorias, proyectos, manuales, etc.) para, en fases posteriores, desbrozar o resumir la información fundamental. Para ello se deberán identificar las barreras existentes para que los procesos de gestión del conocimiento sean fluidos y asumidos por la organización, así como formar y explicar de una manera clara a todos los miembros integrantes, lo que supone un proyecto de GC en mantenimiento, con el fin de motivar y marcar las mayores condiciones para el éxito de su implementación. 
Posteriormente en una segunda fase, se formalizan los procedimientos y estrategias para el soporte del modelo de GC, donde se va transformando lo intangible en visible, para la utilización posterior de un banco común de sustentación del conocimiento, mediante cualquier tipo de herramienta (lo común es una herramienta informática, aunque no tiene porqué ser así), comenzándose a gestionar el conocimiento, superando las barreras detectadas, y clarificando el conocimiento en función de las actividades estratégicas de la empresa. Es en esta fase donde se deben definir las personas que harán las funciones de gestores de conocimiento, cuya misión es dar soporte, coordinación y generar pro-actividad entre todos los miembros de la organización, para llevar el proyecto de GC por una senda o dirección definida en la uniformidad en los procesos fundamentales de generación, transmisión y utilización del conocimiento.

Esta segunda fase requiere un profundo estudio para extraer el conocimiento tácito implícito en el personal operativo de mantenimiento, así como el aligeramiento de la información explícita que existe en la organización, con el fin de articular la plataforma tecnológica que dará soporte al contenedor del conocimiento.

En la tercera fase, se produce el asentamiento y continuidad del sistema de GC, dando soporte a los elementos generadores con la captación del conocimiento estratégico y fortaleciendo los ambientes de aprendizaje y las comunidades de prácticas. El seguimiento debe ser continuo marcando estrategias de incentivos y bonificaciones para la correcta gestión del conocimiento. Cuando se llega a un nivel de difusión de la GC a nivel de la organización de mantenimiento, se producen transformaciones visibles en la forma en que se enfrentan a los problemas, averías y experiencias diarias, produciéndose una mayor eficiencia en los procesos, reduciendo tiempos de actuación, y reduciendo los periodos de acoplamiento de nuevos operarios. El sistema es utilizado como parte fundamental en el auto-aprendizaje de los operarios, teniendo en cuenta los criterios y punto de vista de ellos para tener éxito el sistema.

Para mejorar los procesos de gestión de conocimiento dentro de la actividad de mantenimiento, son adecuados los métodos que se han etiquetado como el de Kaizen, planteándolo como sistemas de planeación de eventos para identificar qué procesos sistemáticamente ocultan desperdicios y eliminarlos, por ejemplo, las actuaciones o reacciones ante averías o fallos críticos en las instalaciones y equipamiento de la empresa (Figura 5).

\section{Conclusiones}

En el presente artículo se han reseñado los dos libros donde se indican los resultados de una investigación, en donde se presenta un modelo para el mantenimiento basado en técnicas de gestión de conocimiento [2], incidente en sus aspectos estratégicos fundamentales que desarrolla en la empresa. Para ello se ha realizado un estudio exploratorio para definir y extraer las características de los procesos que se dan en el desempeño en esta actividad, extrayéndose las barreras y condicionantes con que se encuentran dichos departamentos y los facilitadores 


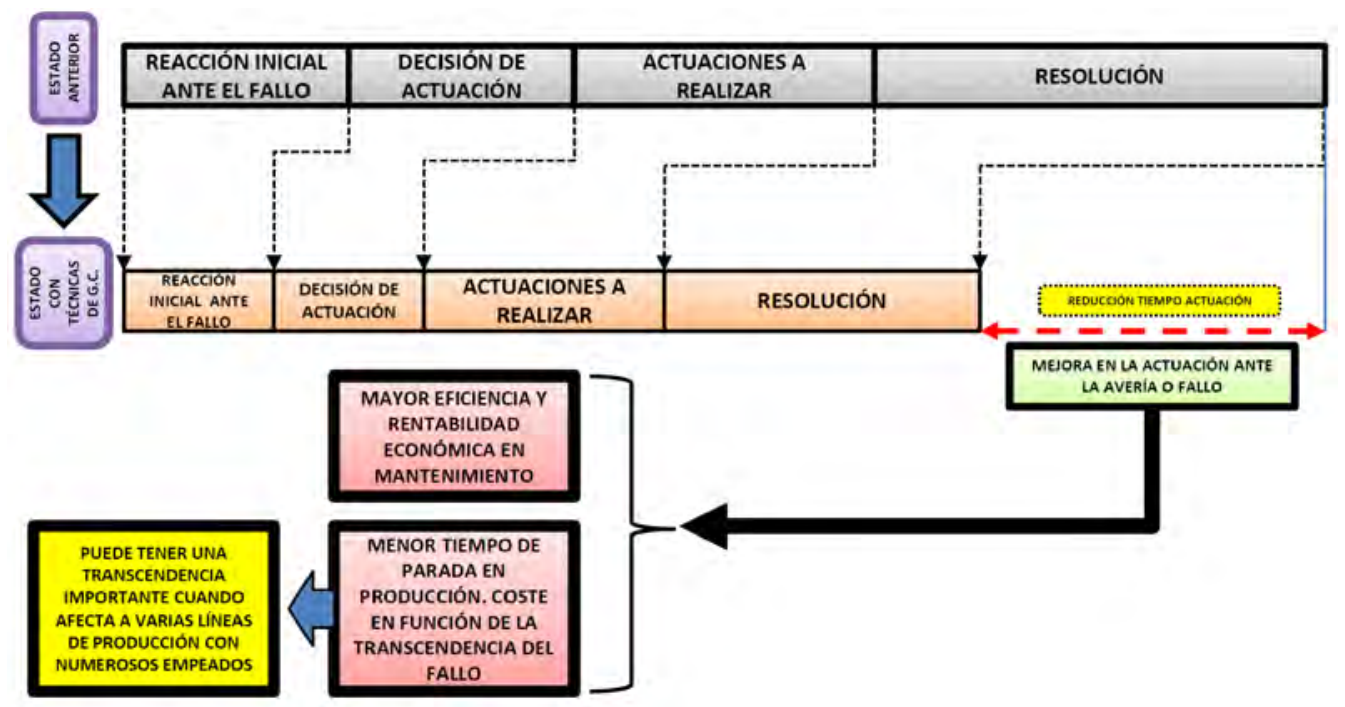

Figura 5. Diagrama Yamazumi con reducción de desperdicios en la actuación ante averías.

fundamentales para vencerlos. Con base en ello, y basado en la literatura existente sobre gestión del conocimiento, se han definido los principios y desarrollado un modelo para su aplicación al mantenimiento. Se ha realizado una investigación de campo en el entorno de una industria del sector alimentario durante un proceso de tres años, obteniendo unos resultados que confirman la bonanza del modelo.

Las aportaciones más relevantes se centran en cómo se muestran las características del uso del conocimiento en mantenimiento en gran parte de las empresas, y la cuantificación de las mejoras que se obtienen con la mejora de esa información y conocimiento estratégico, que normalmente y pese a tener un alto valor intangible, no está custodiado y en poder de la empresa, sino que se encuentra en gran medida en forma tácita entre los operarios de mantenimiento.

En el libro de investigación titulado "La gestión del conocimiento en la ingeniería del mantenimiento industrial: investigación sobre la incidencia en sus actividades estratégicas" [1], se realizó una descripción del estado de la situación y los principios básicos de la gestión del conocimiento y de la ingeniería del mantenimiento, estudiándolo dentro de las áreas de explotación y mantenimiento, con el fin de conocer las barreras y facilitadores, que dicho personal implicado encuentra para que se produzca una adecuada transmisión y utilización de dicho conocimiento fundamental, definiéndose las actividades estratégicas que realizan los departamentos de mantenimiento, y la manera en que repercuten en la empresa.

\section{Referencias}

1. Cárcel Carrasco, Francisco Javier. (2014). "La gestión del conocimiento en la ingeniería del mantenimiento industrial: Investigación sobre la incidencia en sus 
actividades estratégicas". Omnia Science, 2014. ISSN 978-84-941872-7-8. DOI: http://dx.doi.org/10.3926/oms.197.

2. Cárcel Carrasco, Francisco Javier. (2014). "Planteamiento de un modelo de mantenimiento industrial basado en técnicas de gestión del conocimiento". Omnia Science, 2014. ISSN 978-84-941872-8-5. DOI: http://dx.doi.org/10.3926/oms.198. 